\title{
MACROBLOCK-ADAPTIVE RESIDUAL COLOR SPACE TRANSFORMS FOR 4:4:4 VIDEO CODING
}

\author{
Detlev Marpe, Heiner Kirchhoffer, Valeri George, Peter Kauff, and Thomas Wiegand
}

Fraunhofer Institute for Telecommunications - Heinrich Hertz Institute, Image Processing Department Einsteinufer 37, D-10587 Berlin, Germany, [marpe|kirchhof|george|kauff|wiegand]@hhi.fraunhofer.de

\begin{abstract}
Block-based video-coding for 4:4:4 color sampling is extended by an adaptive color space transform. The presented technique enables an encoder to switch between several given color space representations in order to optimize ratedistortion performance. Simulations based on the current draft of the H.264/MPEG4-AVC 4:4:4 extensions demonstrate that our technique provides a rate-distortion performance equal or better than that obtained when using any of the individual color spaces only.
\end{abstract}

Index Terms - Color transforms, video coding, H.264/MPEG4-AVC.

\section{INTRODUCTION}

In contrast to typical consumer applications, many highquality video applications such as professional digital video recording or digital cinema are requiring all three color components to be represented with identical spatial resolution - a representation also known as 4:4:4 color sampling. Moreover, for this kind of applications sample values in each color component of a video signal are expected to be captured and displayed with a precision of more than 8 bits. These specific characteristics are posing new questions and new challenges, especially regarding the choice of an optimal color space representation.

Typically, for both, video capture and display purposes the $R G B$ (red, green, and blue) color space representation can be considered as the natural choice. From a coding point-of-view, however, the $R G B$ domain is often not the optimum color space representation, mainly because for natural source material usually a quite significant amount of statistical dependencies between the $R G B$ components can be observed. Thus, in order to take advantage of these statistical properties, a decorrelating transformation from $R G B$ to some appropriate color space should be applied. Several such color transforms are specified by standardization bodies like, e.g., ITU or SMPTE, where the most relevant of them for video coding purposes are referenced in Annex E of the H.264/MPEG4-AVC video coding standard [1]. The corresponding color spaces are usually denoted by $\mathrm{YCbCr}$ to indicate conversion to a representation consisting of one luma $(Y)$ and two color difference $(\mathrm{Cb}, \mathrm{Cr})$ components.

Apart from the decorrelating properties, RGB-to-YCbCr color transforms are also beneficial in distinguishing be- tween what the human visual system perceives as brightness and as color attributes. Coding in the 4:2:0 color format, as it is mostly used in consumer applications, typically exploits these effects by first transforming to $\mathrm{YCbCr}$ and then subsampling the resulting color difference (i.e., chroma) components both horizontally and vertically by a factor of 2 . In addition, existing video coding standards such as H.264/MPEG4-AVC often provide only restricted tool sets for predictive coding of chroma components relative to those available for the luma component [1].

In high-fidelity video applications, such as currently addressed by a new standardization activity for extending H.264/MPEG4-AVC towards the specification of so-called Advanced 4:4:4 profiles [2], more care has to be taken to ensure a minimum level of distortion across all color components at a given bit rate. As one consequence of these requirements, the current draft of the Advanced 4:4:4 coding architecture [3] specifies all three input components to be treated exactly in the same way as the luma component in the existing High profiles of H.264/MPEG4-AVC [1]. Interestingly, typical coding results produced with the current H.264/MPEG4-AVC 4:4:4 draft design have clearly shown that the effectiveness of a decorrelating color transform in terms of rate-distortion (R-D) performance highly depends on the specific coding conditions as well as the given source characteristics. In many applications, however, both the characteristics of the source and the coding conditions are not known in advance and may also change with respect to the spatial and temporal domain. Consequently, any fixed, a priori chosen color representation may result in an overall suboptimal R-D behavior.

As a solution to this problem, we propose a technique which allows to adapt the color space representation of a 4:4:4 video signal on a block-by-block basis. We further propose to apply this adaptive color space transform to the spatially transformed prediction residual only, which saves computational cost as well as memory bandwidth and, in addition, enables us to apply the color space transform to selected low frequency components only. In this way, it is possible to exclude high frequency components from the application of the color transform in case the given video signal contains a substantial amount of uncorrelated noise across the $R G B$ components as it is, e.g., often the case for film-scanned video sources. 


\section{BACKGROUND AND PROBLEM STATEMENT}

Recently, an interesting candidate for a color transform has been proposed in the context of the H.264/MPEG4-AVC standardization [5]. This color transform maps $R G B$ to the so-called $Y C O C g$ color space, and it has some remarkable properties. First, this color transform has been shown to be capable of achieving a decorrelation that is much better than that obtained by various $R G B$-to- $Y C b C \mathrm{r}$ transforms and which, in fact, is very close to that of the Karhunen-Loève transform (at least, when measured for a representative set of high-quality $R G B$ test images) [5]. Secondly, the transform is reversible in the sense that each original $R G B$ triple can be exactly recovered from the corresponding $Y C o C g$ triple if the color difference components $\mathrm{Co}$ and $\mathrm{Cg}$ are represented with one additional bit accuracy relative to the bit depth used for representing $R G B$, and if furthermore, no information loss in any subsequent coding step is assumed. Thirdly and finally, both the forward and inverse $R G B$-to- $Y C o C g$ transform can be implemented with relatively low complexity since both operations require only a few shift and add operations per triple which, in addition, can be performed inline, i.e., without the need of extra memory apart from one single auxiliary register:

$$
\begin{aligned}
C o & =R-B \\
t & =B+(C o \gg 1) \\
C g & =G-t \\
Y & =t+(C g \gg>1)
\end{aligned} \Leftrightarrow \quad \begin{aligned}
t & =Y-(C g \gg>1) \\
B & =t-(C o>>1) \\
R & =B+C o
\end{aligned}
$$

The " $>>$ "-operator in (1) denotes the bitwise right shift operator. Note that the transform steps have to be performed in the order from top to bottom in (1) to guarantee that the memory locations for $G, B$, and $R$ can be re-used for $\mathrm{Y}, \mathrm{Cg}$, and $\mathrm{Co}$, respectively, and vice versa.

In order to evaluate the R-D performance of the $R G B$-to$Y C O C g$ color transform for the intended high-quality $4: 4: 4$ video coding applications, we have tested a variety of natural video material ranging from film-scanned sequences to film material that was directly captured from a high-quality 3-CCD camera [6]. For these coding simulations, we have used an implementation of the current draft Advanced 4:4:4 profiles [3], driven in intra-only coding mode. Fig. 1 demonstrates the characteristic output of our experiments, where the red and green curves represent the R-D performance for encoding the same source in the $R G B$ and $Y C o C g$ domain, respectively. As can be seen from Fig. 1, at relatively low bit rates the $Y C O C g$ representation performs significantly better than the corresponding $R G B$ representation, whereas $R G B$-based encoding leads to an increasingly better performance when moving towards higher bit rates.

This phenomenon can be mainly attributed to the fact that most natural video sources contain a relatively high amount of signal-independent, uncorrelated noise with varying noise power over all three primary channels. Typically, the blue $(B)$ channel exhibits the most dominant noise signal which, when transformed to the $Y C O C g$ domain, gets spread over all three resulting components. As a result, the overall noise power in the $Y C O C g$ representation is increased relative to that in the $R G B$ domain. This, in turn, leads to the observed degradation of coding efficiency in the medium to high bitrate range where more and more noise components typically are supposed to survive the quantization process. As a further consequence, a cross-over region can be observed, indicating a suboptimal R-D performance of both fixed alternative color space representations relative to the R-D envelope, as illustrated by the dashed curve in Fig. 1. Note that in either case, for encoding in a single color space representation, one can only move along one or the other R-D curve.

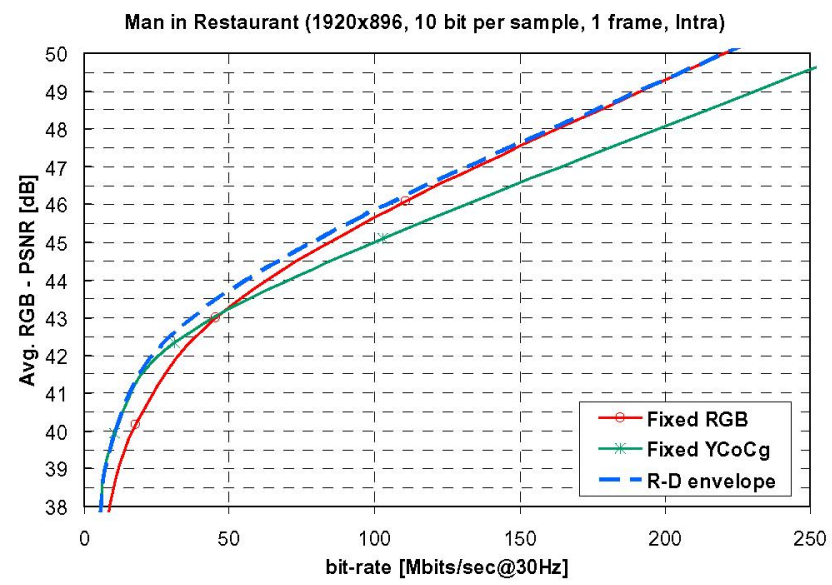

Fig. 1 - $R-D$ curves for intra-only coding of a single picture of a filmscanned test sequence using the two fixed color space representations $R G B$ and $Y C o C g$. The dashed curve, partly covering the $R-D$ curves for $R G B$ and $Y C O C g$-based encoding shows the corresponding $R-D$ envelope.

In general, the effectiveness of a color transform in terms of decorrelation will also depend on other source characteristics such as, e.g., given by the degree of color saturation or the amount of texture information and its alignment across the R, G, and B components. Typically, this kind of statistical properties are often subject to changes both within a given picture and from picture to picture.

Based on these observations, the fundamental problem to be addressed in this paper is how to supply a reasonable collection of candidate color spaces and how to choose between these color space representations in such a way that an optimal R-D performance is always guaranteed, independently from the chosen coding conditions and the specific source characteristics.

\section{ADAPTIVE COLOR SPACE TRANSFORM}

\subsection{Basic idea}

Our present approach is based on the finding that a picture or a series of pictures can be encoded more efficiently when each picture is partitioned into smaller regions. Each region then carries the picture information either in a primary color 
space representation (e.g., $R G B$ ) or in one of several secondary color space representations (e.g., $Y C o C g$ ). In this way, the color transform such as, e.g., $R G B$-to- $Y C o C g$, can be applied in a spatially adaptive way by taking into account the varying statistical properties of the source. The decision which color space representation to use is made by the encoder and signaled to the decoder as side information.

\subsection{Simple in-loop decorrelating transform}

Additionally to the $Y C O C g$ color space we further utilize a very simple transform from $R G B$ to another "color" space that we have abbreviated by $G r_{B} r_{R}$ and that is given by

$$
\left[\begin{array}{c}
G \\
r_{B} \\
r_{R}
\end{array}\right]=\left[\begin{array}{ccc}
1 & 0 & 0 \\
-1 & 1 & 0 \\
-1 & 0 & 1
\end{array}\right]\left[\begin{array}{c}
G \\
B \\
R
\end{array}\right]
$$

According to this transform, $G$ is not modified and acts as a predictor for $B$ and $R$. Because of the vanishing off-diagonal elements in the second and third column of the transform matrix in (2), potential noise components cannot be propagated from the blue or red channel to any of the other channels. Furthermore, since the matrix on the right hand side of (2) is a (lower) triangular matrix, it can be applied to a triplet of primaries containing the quantized $G$ component instead of the original $G$ signal. This "closed-loop" processing enables the encoder to use exactly the same quantized $G$ signal for prediction (of $B$ and $R$ ) as the decoder which, in turn, has the advantage that the quantization noise of $G$ will be taken into account for the transformed $r_{B}$ and $r_{R}$ components.

\subsection{Frequency-selective usage of color transforms}

Typically, for high-frequency spatial transform coefficients the power of the noise signal as resulting, e.g., from film grain or from a $\mathrm{CCD}$, relative to the power of the wanted signal can expected to be quite dominant. Thus, to prevent the propagation of those noisy coefficients from the G component to the corresponding coefficients of the $r_{B}$ and $r_{R}$ components, we introduce a frequency-selective application of the corresponding color transform by restricting its application to spatial low frequency coefficients in a way as further described below.

\subsection{Integration into H.264/MPEG4-AVC}

Our proposed approach has been integrated into the current draft design of H.264/MPEG4-AVC Advanced 4:4:4 profiles. By doing so, several design decisions with respect to a specific incarnation of the rather general idea have been made. First, the size of blocks for the switchable color space has been chosen to be aligned with the fixed macroblock (MB) partitioning (of $16 \times 16$ samples for each color component) in H.264/MPEG4-AVC. This choice was also motivated by the desire to achieve a reasonable trade-off between the additional side information needed to indicate the choice of the color space and the coding gain obtained by spatially adapting the color space representation in a most accurate way.

The second important design decision is related to the specific application of the color space transform itself. Mathematically, there is no difference in applying a color transform before forming and applying the prediction, or vice versa, provided the same (linear) prediction operator is applied to all three color components ${ }^{1}$, as it is the case in the current draft H.264/MPEG4-AVC 4:4:4 design [3]. However, operating in the original domain would imply that the color transform has to be applied to the reference signal as well, whenever required by the encoder's choice. This, together with the need for an increased bit depth in the $\mathrm{Co}$ and $C g$ or $r_{B}$ and $r_{R}$ representation, would have led to a considerable overhead in computational cost as well as memory bandwidth when compared to the relatively resource-friendly way of applying the color space transform to the prediction residual.

Consequently, we decided to operate on the residual signal and called our approach the macroblock-adaptive residual color transform (MB-ARCT). Moreover, in our proposed MB-ARCT approach we have reversed the order of application of spatial transforms and the proposed adaptive color space transform. This commutation is necessary to accomplish the frequency-selective application of the $R G B$ to- $G r_{B} r_{R}$ color space transform. For achieving the desired amount of adaptivity, our simulations have shown that it is sufficient to choose between the following three color space representations per macroblock:

- $\boldsymbol{R} G \boldsymbol{B}$ : no coefficient of the corresponding macroblock is color transformed.

- YCoCg: all coefficients of the corresponding MB are transformed to $Y C o C g$ according to (1).

- $G r_{B} r_{R}$ : only $1 / 4$ of coefficients along the zig-zag scan, starting from the DC in a spatially transformed block are transformed to $G r_{B} r_{R}$ according to (2).

The choice of the encoder (as performed along the process described below) is signaled by means of two separate flags at the macroblock layer. This implies a maximum overhead of 2 bits/MB with a considerably lower effective rate, especially in case of using the CABAC entropy coding mode [1]. The modeling part of $\mathrm{CABAC}$ has been extended to include an additional adaptive probability models for each of those flags.

\subsection{R-D optimized color space selection process}

In our specific encoder implementation, the MB-adaptive choice of the residual color transform is performed as a straightforward extension of the usual mode decision process based on a Lagrangian cost function $J=D+\lambda R$ [7]. For each given macroblock and for each of the three candidate color space representations of the prediction residual, a

\footnotetext{
${ }^{1}$ In a mathematically strict sense this is, however, only true if any rounding or clipping operations are neglected.
} 

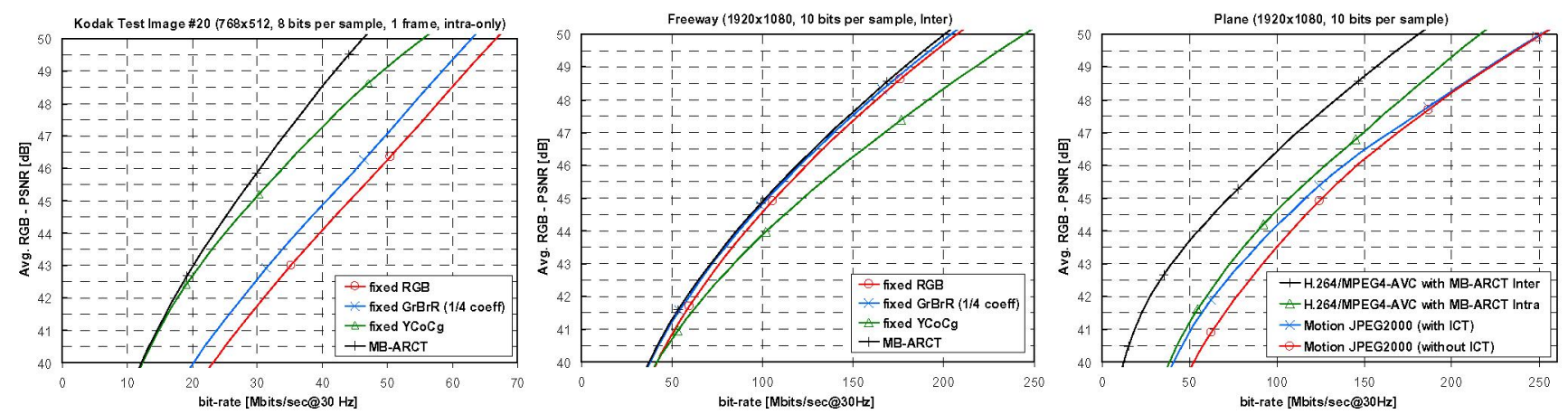

Fig. 2 - Left and middle: R-D curves comparing fixed $R G B, G r_{B} r_{R}$, and $Y C o C g$ encoding with the MB-ARCT method for the intra and inter case, respectively. Right: R-D comparison of H.264/MPEG4-AVC using MB-ARCT (both for intra and inter coding) and Motion JPEG2000.

candidate prediction mode is selected employing the same strategy as described in [7]. Based on this preselection, the underlying color space representation with the lowest overall Lagrangian cost $J$ is finally chosen. Note that for the distortion measurement $D$ of the corresponding Lagrangian $J$, the averaged mean squared error over all three components in the primary color representation $(R G B)$ has been used.

\section{EXPERIMENTAL RESULTS}

We have implemented our proposed approach as described in the previous section on top of an implementation of the current draft Advanced 4:4:4 profiles [3]. In Fig. 2, coding results are shown comparing the R-D performance of our proposed MB-ARCT method with coding schemes based on fixed color space representations. The left graph of Fig. 2 demonstrates intra-coding results for a particular still image which were obtained by using H.264/MPEG4-AVC with and without MB-ARCT. For this 24 bit $R G B$ image of the Kodak test image set [8], MB-ARCT actually achieved an overall R-D gain compared to the best performing fixed color space representation due to its ability of spatially adapting the residual color representation to the varying source characteristics as well as the varying coding conditions. As can be seen from the corresponding graph, substantial R-D gains of up to $1.5 \mathrm{~dB}$ average $R G B$ PSNR have been observed in favor of MB-ARCT.

The R-D graph in the middle of Fig. 2 shows some simulation results for the inter case, i.e., the case of using motion-compensated prediction with both $P$ and $B$ pictures. We observed a similar R-D behavior as in the intra-only case with the general trend of smaller overall R-D gains, as exemplified in Fig. 2 (middle) for the "Freeway" Viper sequence. An R-D performance comparison of our MBARCT enhanced H.264/MPEG4-AVC implementation with Motion-JPEG2000 [9] was made as well by using an JPEG2000 software implementation [10]. Fig. 2 (right) illustrates a sample of the corresponding coding results. As can be seen from the corresponding R-D graph, H.264/MPEG4-AVC intra-only coding with MB-ARCT performs better than Motion-JPEG2000, even if the latter is using the JPEG2000-specific irreversible color transform
(ICT). In most of our investigated cases, H.264/MPEG4AVC inter coding delivered a substantially improved R-D performance relative to H.264/MPEG4-AVC intra-only coding both with and without using MB-ARCT, as illustrated for the former case in Fig. 2 (right).

\section{CONCLUSIONS}

We have presented a relatively simple but rather efficient approach to resolve the critical issue of selecting an appropriate color space representation in H.264/MPEG4-AVC Advanced 4:4:4 coding profiles. For that purpose, we have introduced an adaptive approach for selecting between a number of given color space representations of the prediction residual on a block-by-block basis. Our coding results have shown that by using the proposed macroblock-adaptive residual color transform approach, it is ensured to always achieve the same or better coding efficiency than by using any of the fixed color space representations at choice. In particular, we have demonstrated that by using the frequency-selective $R G B$-to- $G r_{B} r_{R}$ transform in addition to the $R G B$ and $Y C O C g$ representations, significant coding gains can be achieved for some representative video sources.

\section{REFERENCES}

[1] ITU-T Rec. H.264 \& ISO/IEC 14496- 10 AVC, "Advanced Video Coding for Generic Audiovisual Services," Version 3, 2005.

[2] T. Suzuki et al., "Justification for new 4:4:4 video coding profile(s)", ISO/IEC JTC I/SC 29/WG 11, N7313, July 2005.

[3] H. Yu, "Draft Text of H.264/AVC Amendment 2", ISO/IEC JTC 1/SC 29/WG 11, ITU-T Q6/SG16, JVT-Q205r1, Oct. 2005.

[4] H. Yu and L. Liu, "Advanced 4:4:4 Profile for MPEG4Part10/H.264," ISO/IEC JTC 1/SC 29/WG 11, ITU-T Q6/SG16, JVTP017, July 2005.

[5] H. Malvar and G. J. Sullivan, "YCoCg-R: A Color Space with RGB Reversibility and Low Dynamic Range", ISO/EC JTC1/SC 29/WG 11, ITU-T Q6/SG16, JVT-I014, July 2003.

[6] Thomson Grass Valley Viper FilmStream Camera, online: http://www.thomsongrassvalley.com/products/cameras/viper/

[7] K.-P. Lim, G. Sullivan, and T. Wiegand, "Text Description of Joint Model Reference Encoding Methods and Decoding Concealment Methods", ISO/IEC JTC 1/SC 29/WG 11, ITU-T Q6/SG16, Document JVT-N046r1, Jan. 2005.

[8] Kodak test image set, online: http://r0k.us/graphics/kodak/index.html

[9] ITU-T Rec. T.802 \& ISO/IEC 15444-3 Motion JPEG2000, 2002.

[10] Kakadu software online: http://www.kakadusoftware.com 